\title{
On the relaxation dynamics of diluted antiferromagnets
}

\author{
M. Staats*, U. Nowak, K.D. Usadel \\ Theoretische Physik und SFB 166, Gerhard-Mercator-Universität-Gesamthochschule Duisburg, 47048 Duisburg, Germany
}

\begin{abstract}
The phase diagram of a diluted Ising antiferromagnet in an external magnetic field has a disordered phase for sufficiently large values of this field and for low temperatures, in which the system's equilibrium state is a domain state. We study the relaxation dynamics out of this state after switching off the magnetic field, for two- and three-dimensional diluted antiferromagnets using Monte Carlo Simulation techniques. We measure the decay of the initial magnetization carried by the domain state. The data can be described by a unique law for a wide range of temperatures, magnetic fields and dilutions in two and three dimensions.
\end{abstract}

Keywords: Dilute antiferromagnet; Random fields; Disordered systems; Magnetization - decay; Relaxation - magnetic

It is generally accepted that the dynamics of phase ordering in systems with quenched disorder is driven by thermal activation following the Arrhenius law $t=$ $\tau \exp (\delta E / T)$, where $t$ is the time necessary for a physical process that has to overcome energy barriers of the height $\delta E$ and $\tau$ is a microscopic time scale of the system, which is expected to be of the order 1 Monte Carlo step in a simulation. This leads to a natural scaling variable of $T \ln (t / \tau)$. In theoretical works the focus is usually laid on some characteristic length scale of the system such as the average domain size [1]. However, it is not always clear in which way physical quantities can be related to such length scales because the complete distribution of domain sizes influences them. In experiments usually the homogeneous magnetization or susceptibilities are measured $[2,3]$. Therefore, in this contribution we focus mainly on the magnetization.

To study the dynamics of phase ordering in strongly disordered systems, we simulated two- and three-dimensional diluted antiferromagnets in an external field (DAFF) by the Monte Carlo method. The Ising-Hamiltonian is given by

$$
\mathscr{H}=-J \sum_{\langle i j\rangle} \varepsilon_{i} \varepsilon_{j} \sigma_{i} \sigma_{j}-B \sum_{i} \varepsilon_{i} \sigma_{i}
$$

\footnotetext{
* Corresponding author. Fax: + 49203379 2965; e-mail: michael@thp.uni-duisburg.de.
}

where the summation runs over the nearest neighbors in a square $(d=2)$ and cubic $(d=3)$ lattice, respectively. The interaction $J$ is set to -1 . The dilution is given by the quenched variables $\varepsilon_{i}$, which are 0 with a probability $p$ and 1 otherwise.

The DAFF is expected to be in the same universality class as the random field Ising model [4]. Therefore, in $d=2$ the system is antiferromagnetically ordered only for $B=0$ below the critical temperature $T_{\mathrm{c}}$ [5]. In $d=3$ there is long-range antiferromagnetic order also for external fields $B>0[2,3]$. In both $d=2$ and $d=3$ the system is in a domain state for sufficiently large $B$. The domain state is characterized by a wide distribution of fractal domains [6]. Experimental realizations of this model are e.g. $\mathrm{Fe}_{1-p} \mathrm{Zn}_{p} \mathrm{~F}_{2}(d=3)$ and $\mathrm{Rb}_{2} \mathrm{Co}_{1-p} \mathrm{Mg}_{p} \mathrm{~F}_{4}$ $(d=2)$, respectively [7].

We prepare the system in an external field and at a low temperature, where the equilibrium state of the system is a domain state. To achieve this we performed a large number of Monte Carlo updates starting from a completely disordered spin state, until the homogeneous magnetization does not change significantly any more. Due to the slow dynamics, the system will not have reached thermal equilibrium but this is not important, the only important aspect here is that the system is in a typical domain state. After this preparation, the external field is switched off and the system will relax into its long-range ordered ground state with zero magnetization. The corresponding relaxation of the magnetization 


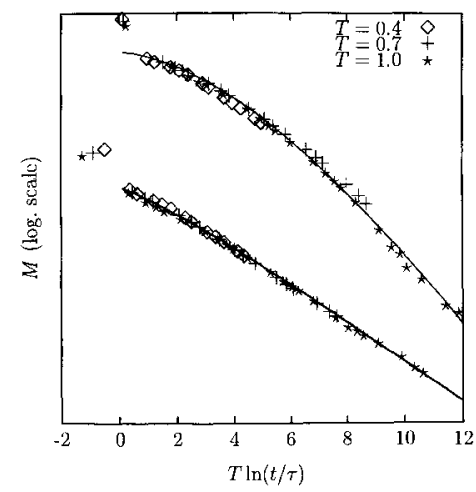

Fig. 1. Magnetization versus scaled time for dilutions $p=0.35$ (upper curve) and $p=0.25$ (lower curve). The solid lines are plots of the scaling functions. (Data for $p=0.25$ are shifted downwards to avoid intersections.)

is measured for different temperatures. For each value of $p$ and $T$ we simulated 20 runs (in $d=2$ ) with different realizations of the dilution and averaged over the results. The lattice size is $400 \times 400$ spins in $d=2$ and $50 \times$ $50 \times 50$ in $d=3$ with periodic boundary conditions. Most of the simulations were done for an initial field of $B=2.0$, but also other values were considered to check for a field dependency of the results. Spins are updated in a random sequential way with the heat-bath algorithm.

The analysis of the data was done in two steps. First, we consider for fixed external field $B$ and fixed dilution $p$ all data for different temperatures. If $T \ln (t / \tau)$ is the correct scaling variable all these data collapse on the same curve by a suitable choice of $\tau$. Fig. 1 , in which data for different values of $p$ are displayed, shows that this works very well. Since the exact value of $\tau$ is unknown, it is important to determine its value from measurements, otherwise a determination of the correct decay law (see below) is extremely difficult due to the slow increase of $\ln (t / \tau)$. Our method allows the determination of the correct value of $\tau$ without further assumptions and without fitting other parameters at this stage of the analysis.

Having established that the decaying magnetization scales with $T \ln (t / \tau)$ we now discuss its scaling function. It is not expected that the magnetization decays as a simple power of the scaling length as does presumably the characteristic domain size since there is no obvious relation between domain size and magnetization. In Ref. [8] we showed for a slightly different situation, i.e. the decay of long-range antiferromagnetic order after switching on an external field, the magnetization approaches its saturation value according to

$M(t) \sim \mathrm{e}^{-b(T \ln (t / \tau))^{y}}$.
Table 1

Results for various dilutions and for $d=2$ and $d=3$

\begin{tabular}{|c|c|c|c|c|c|c|}
\hline \multirow[b]{2}{*}{$p$} & \multicolumn{5}{|l|}{$d=2$} & \multirow{2}{*}{$\begin{array}{l}d=3 \\
0.5\end{array}$} \\
\hline & 0.15 & 0.2 & 0.25 & 0.3 & 0.35 & \\
\hline$y$ & $\approx 1$ & $\approx 1$ & 1.15 & 1.3 & 1.5 & 1.2 \\
\hline$\tau$ & 5 & 3 & 1.8 & 1 & 0.4 & 1.2 \\
\hline
\end{tabular}

We expect a similar time dependence for the decay of the magnetization in the present case. This time dependence for the remnant magnetization has also been found in spin glasses, e.g. Ref. [9]. Note that experimental results [10] as well as earlier simulations [11] for three-dimensional systems can also be fitted by this function. With this function a fit of our time scaled data can be done as shown in Fig. 1. Our results are displayed in Table 1.

We find that $y$ and $\tau$ depend on the dilution of the system. For small dilutions we cannot distinguish between a pure power-law decay $(y=1)$ and a modified power law with $y \neq 1$. For larger dilutions it is obvious that a simple power law cannot fit the data, but the scaling with $T \ln (t / \tau)$ seems to be a universal feature of the DAFF, independent of the spatial dimension $d$ and of the external field $B$, at which the domain state is prepared. It is unclear whether the dilution dependency of the exponent $y$ is due to a non-universal behavior or due to a crossover to a different dynamics near the percolation threshold.

This work was in part supported by the Deutsche Forschungsgemeinschaft through Sonderforschungsbereich 166 .

\section{References}

[1] D.S. Fisher, in: Phase Transition and Relaxation in Systems with Competing Energy Scales, Kluwer, Dordrecht, 1993 , p. 1.

[2] J.Z. Imbrie, Phys. Rev. Lett. 53 (1984) 1747.

[3] J. Bricmont, A. Kupiainen, Phys. Rev. Lett. 59 (1987) 1829.

[4] S. Fishman, A. Aharony, J. Phys. C 12 (1979) L729.

[5] M. Aizenman, J. Wehr, Phys. Rev. Lett. 62 (1989) 2503.

[6] J. Esser, U. Nowak, K.D. Usadel, Phys. Rev. B. 55 (1997) 5866.

[7] W. Kleemann, Int. J. Mod. Phys. B 7 (1993) 2469.

[8] U. Nowak, J. Esser, K.D. Usadel, Physica A (1996) 40.

[9] J.L. van Hemmen, A. Sütö, Z. Phys. B 61 (1985) 263.

[10] S.-J. Han, D. Belanger, W. Kleemann, U. Nowak, Phys. Rev. B 45 (1992) 9728.

[11] S.-J. Han, D.P. Belanger, Phys. Rev. B 46 (1992) 2926. 